\title{
Assessment of Client/Center Admimistrative Factors That Affect Nurses' Utilization of Partograph in Primary/Secondary Health Care Facilities in Enugu Metropolis
}

\author{
Nwaneri $\mathrm{A}^{1,}$ Ndie E $\mathrm{C}^{3}$,Ehiemere $\mathrm{I}^{1}$, Okafor $\mathrm{E}^{1}$,Ezenduka $\mathrm{P} \mathrm{O}^{2}$, and Emeh $\mathrm{A}^{3}$ \\ ${ }^{(1)}$ Department of Nursing science, University of Nigeria, Enugu Campus, Nigeria \\ ${ }^{(2)}$ Department of Nursing Science, Nnamdi Azikiwe University, Nnewi Campus, Nigeria \\ ${ }^{(3)}$ Department of Nursing Science, Ebonyi State University, Abakaliki, Nigeria
}

\begin{abstract}
This study aimed assessing the contribution of clients and care center administrative to the problems nurses are having in the utilization of partograph in the labour wards in Enugu metropolis. Convenient sampling technique was use to select seven health care facilities. Total of 126 nurse/midwives who work in their labour rooms were used for the study. Self-structured questionnaire was used as instrument for data collection. The results show that woman reporting late in labour $(73.8 \%)$ ), woman with disease condition such as severe pregnancy induced hypertension (74.6\% ), or confirmed cephalo-pelvic disproportion (55\%) and woman with antepartum haemorrhage which contributed to poor utilization of partograph by nurses in labour room. The results also show that the care center administration do not provide the needed material for the nurses to work with. It was the recommended the though client present late and in most cases present with complications, nurses must do their work using partograph and following obstetric emergency protocol and that hospital administration should provide the needed materials to enable nurses do their work.
\end{abstract}

Keywords; Client factors, center administration factors, nurses' utilization, partograph, health care facilities and Enugu metropolis

\section{Introduction}

The majority of the maternal deaths and complications attributable to obstructed and prolonged labour could be prevented by cost effective and affordable health intervention like the use of partograph (Magon, 2011). The partograph is a tool developed by Philpott in 1971 and was later modified by the World Health Organization (Mathibe-Neke, Lebeko \& Motupa, 2013).

A partograph is one of the valuable appropriate technologies in use for improved monitoring of labour progress and maternal and fetal well- being. It is an important tool for managing labour that enables the midwives, nurses and doctors to record their examination findings on a standardized form which generates a pictorial overview of labour progress and allows for early identification and diagnosis of pathological labour (Fawole, Adekanle \& Hunyinbo, 2010). Its' use is critical in preventing maternal and perinatal morbidity and mortality and therefore has applicability in developed and developing world settings (Fawole, Hunyinbo \& Adekanle, 2008).

A partograph has clear demarcations which if arrived at or exceeded clearly, indicates the need to address existing or imminent complications like poor progress of labour, prolonged labour, fetal distress and in the worst cases, obstructed labour and ruptured uterus. It also aids early detection of obstructed labour and other related complications such as postpartum haemorrhage, ruptured uterus, puerperal sepsis and obstetric fistula (Mathai, 2010).

Despite the World Health Organization advocating and recommending that the partograph be compulsorily used in monitoring all labour process since 1994, it was and is still reported to be in a limited extent in the developing countries like Nigeria (Maternal and Neonatal Health (M.N.H),2012). This is especially so in primary and secondary health care facilities where most of the deliveries take place (Okechukwu, Adesegun \&Wiyi, 2013). A report from a collaborative workshop held in Japan in 2012 on the education of midwives revealed that midwives might not receive adequate training to enable them to work as competent and confident practitioner (Walker, 2012).

Literature has indicated several reasons why the partograph is either not used or not used properly due to non-availability of partograph charts in labour ward and poor knowledge of the partograph that affecting its use (Fawole, Adekanle and Hunyinbo 2010 and Umezulike, Onah and Okaro 2009) Since the utilization of the partograph has been met with difficulties especially in primary/secondary health care facilities, it becomes necessary to look into those factors emanating from the patients and the care setting that influence the utilization of the partograph among nurses and midwives in selected primary/ secondary health care facilities in Enugu urban. The study aimed at assessing client/care centered administrative factors that Impede/promote utilization of the partograph by nurses in primary/secondary health care facilities in Enugu metropolis. 


\section{Methodology}

Descriptive survey design was used for this study. The health institutions selected by convenience sampling method were Poly Sub-District hospital Asata, St Patrick's hospital Asata, Mother of Christ Specialist hospital Ogui, Balm of Gilead hospital Maryland, Redeemer Maternity Abakpa, Iji Nike cottage hospital and Uwani cottage hospital. The subjects of study comprise of 126 nurses/ midwives working in the labour wards of these seven selected health care facilities in Enugu urban. Due to the small population of the nurses/ midwives working in labour wards of the selected health care facilities, the whole population was selected for the study. No sampling procedure therefore was carried out. However, involvement in the study was dependent on the following inclusion criteria of registered nurses/midwives practicing midwifery in labour wards of the selected health facilities and willingness to participate in the study. The instrument for data collection was a questionnaire drawn strictly based on extensive literature search on partograph in order to fit the objectives of the study by the researchers.

Pilot study was conducted for the reliability of the instrument using test- retest method distributed to registered nurses/ midwives working in labour wards at Good Shepherd Hospital Uwani that had similar characteristics set up as the selected health care facilities of study. The test-retest reliability using the Cronbach's alpha coefficient obtained was 0.773 . This coefficient being greater than 0.7 indicate that the reliability of the test instrument is very strong. Thus the reliability of the research instrument was established. Permission to carry out the study in the selected health care facilities was obtained from the heads of the selected health care facilities in Enugu urban, since they did not have ethical committees. The researchers then approached the subjects, and obtained verbal informed consents from them. The copies of the questionnaire were distributed to each nurse/midwife in the selected health care facilities through by the researchers at the beginning of the shift and collected back before the end of the shift. A total of 126 copies were recovered. Data collected were presented in tables and charts. The responses to each question were tallied and analyzed using descriptive statistics ranging from mean-scores to standard deviation. They were grouped according to study objectives. The data were analyzed using the IBM Statistical Package for Social Sciences (SPSS) version 20.

\section{Results}

Table 1: Client factors that determine non - utilization of the Partograph $\quad(\mathbf{n}=\mathbf{1 2 6})$

\begin{tabular}{|l|l|l|l|l|l|}
\hline Response & SA (\%) & A (\%) & D (\%) & SD (\%) & Mean(SD) \\
\hline $\begin{array}{l}\text { Woman reporting late in labour at 9cm to 10cm cervical } \\
\text { dilatation }\end{array}$ & $93(73.8)$ & $27(21.4)$ & $6(4.8)$ & $0(0)$ & $3.69(0.56)$ \\
\hline Woman for elective ceasarean section & $94(74.6)$ & $22(17.5)$ & $9(7.1)$ & $1(0.8)$ & $3.66(0.65)$ \\
\hline Woman for emergency caesarean section & $70(55.6)$ & $23(18.3)$ & $23(18.3)$ & $10(7.9)$ & $3.21(1.01)$ \\
\hline $\begin{array}{l}\text { Woman with disease condition such as severe } \\
\text { pregnancy induced hypertension, eclampsia or } \\
\text { confirmed cephalo-pelvic disproportion }\end{array}$ & $63(50.0)$ & $29(23.0)$ & $33(26.2)$ & $1(0.8)$ & $3.22(0.87)$ \\
\hline $\begin{array}{l}\text { Woman with gestational age less than 30weeks } \\
\text { Woman with antepartum haemorrhage }\end{array}$ & $51(40.5)$ & $18(14.3)$ & $42(15.9)$ & $15(11.9)$ & $2.83(1.09)$ \\
\hline
\end{tabular}

Overall mean

Table 1 revealed the client factors that determine the non-utilization of the partograph. 93(73.8\%) of the respondents strongly agreed that woman reporting late in labour at $9 \mathrm{~cm}$ to $10 \mathrm{~cm}$ cervical dilatation determines their non-utilization of the partograph, 27(21.4\%) agreed to this factor determining their nonutilization of the partograph, $6(4.8 \%)$ disagreed while none (0\%) strongly disagreed that this factor determines their non-utilization of the partograph. The mean response for this factor was 3.69(0.56). 94(74.6\%) of the respondents strongly agreed that woman for elective ceasarean section determines their non-utilization of the partograph, 22(17.5\%) agreed that this factor determines their non-utilization of the partograph, 9(7.1\%) disagreed to this factor determining their non-utilization of the partograph while $1(0.8 \%)$ strongly disagreed. The mean response for this factor was 3.66(0.65). 70(55.6\%) of the respondents strongly agreed that woman for emergency caesarean section determines their non-utilization of the partograph, 23(18.3\%) each agreed and disagreed to this factor determining their non-utilization of the partograph while 10(7.9\%) strongly disagreed that this factor determines their non-utilization of the partograph. The mean response for this factor was $3.21(1.01) .63(50 \%)$ of the respondents strongly agreed that woman with disease condition such as severe pregnancy induced hypertension, eclampsia or confirmed cephalo-pelvic disproportion determines their nonutilization of the partograph, $29(23 \%)$ agreed, 33(26.2\%) disagreed that this factor determines their nonutilization of the partograph while $1(0.8 \%)$ strongly disagreed. Their mean response for this factor was $3.22(0.87) .51(40.5 \%)$ of the respondents strongly agreed that woman with gestational age less than 30 weeks 
determines their non-utilization of the partograph, 18(14.3\%) agreed to this factor, $42(15.9 \%)$ disagreed while $15(11.9 \%)$ strongly disagreed o this factor determining their non-utilization of the partograph. The mean response for this factor was 2.83(1.09). 76(60.3\%) of the respondents strongly agreed that woman with antepartum haemorrhage determines their non-utilization of the partograph, 21(16.7\%) agreed to this factor determining their non-utilization of the partograph, 28(22.2\%) disagreed that this factor determines their nonutilization of the partograph while $1(0.8 \%)$ strongly disagreed. The mean response for this factor was $3.37(0.85)$.

The mean value of the factors was greater than the decision rule of 2.50. The factors include woman reporting late in labour at $9 \mathrm{~cm}$ to $10 \mathrm{~cm}$ cervical dilatation (3.69), woman for elective ceasarean section (3.66), woman for emergency caesarean section (3.21), woman with disease condition such as severe pregnancy induced hypertension, eclampsia or confirmed cephalo-pelvic disproportion (3.22), woman with gestational age less than 30weeks (2.83) and woman with antepartum haemorrhage (3.37). In the overall, the client factors for the non utilization of the partograph are with mean value of 3.33

Table 2: Administrative factors that determine non - utilization of the Partograph

\begin{tabular}{|l|l|l|l|l|l|}
\hline Response & SA $(\%)$ & A $(\%)$ & D $(\%)$ & SD $(\%)$ & Mean(SD) \\
\hline $\begin{array}{l}\text { Non-availability of resources such as observation tools, for } \\
\text { example sphygnomanometer, fetoscopeetc }\end{array}$ & $62(49.2)$ & $50(39.7)$ & $7(5.6)$ & $7(5.6)$ & $3.32(0.82)$ \\
\hline Non availability of partograph chart in the labour ward & $84(66.7)$ & $41(32.5)$ & $0(0)$ & $1(0.8)$ & $3.65(0.53)$ \\
\hline Shortage of staff & $69(54.8)$ & $43(34.1)$ & $14(11.1)$ & $0(0)$ & $3.44(0.69)$ \\
\hline $\begin{array}{l}\text { Ineffective and inadequate supervision from immediate boss } \\
\text { Lack of recognition /motivation of workers }\end{array}$ & $25(19.8)$ & $61(48.4)$ & $38(30.2)$ & $2(1.6)$ & $2.87(0.74)$ \\
\hline $\begin{array}{l}\text { Lack of standard institutional guideline/ protocol on the use } \\
\text { of partograph }\end{array}$ & $65(51.6)$ & $39(31.0)$ & $6(4.8)$ & $16(12.7)$ & $3.21(1.02)$ \\
\hline $\begin{array}{l}\text { Lack of training of staff through organization of seminars/ } \\
\text { workshops on partograph in the health care facilities }\end{array}$ & $\begin{array}{l}102(81.0 \\
\text { ( })\end{array}$ & $24(19.0)$ & $0(0)$ & $0(0)$ \\
\hline
\end{tabular}

Table 2 revealed the administrative factors that determine the non-utilization of the partograph. $62(49.2 \%)$ of the respondents strongly agreed that non-availability of resources such as observation tools, for example sphygnomanometer, fetoscope, determines their non-utilization of the partograph, 50(39.7\%) agreed that this factor determines their non-utilization of the partograph while 7(5.6\%) each disagreed and strongly disagreed to this factor determining their non-utilization of the partograph. The mean response for this factor was $3.32(0.82) .84(66.7 \%)$ of the respondents strongly agreed that non availability of partograph chart in the labour ward determines their non-utilization of the partograph, 41(11.1\%) agreed to this factor determining their non-utilization of the partograph, none $(0 \%)$ disagreed, while $1(0.8 \%)$ strongly disagreed that this factor determines their non-utilization of the partograph. The mean response for this factor was 3.65(0.53). 69(54.8\%) of the respondents strongly agreed that shortage of staff determines their non-utilization of the partograph, $43(34.1 \%)$ agreed to this factor, $14(11.1 \%)$ disagreed to this factor determining non-utilization of the partograph while none $(0 \%)$ strongly disagreed. The mean response for this factor was $3.44(0.69 \%)$. 25(19.8\%) of the respondents strongly agreed that ineffective and inadequate supervision from immediate boss determines their non-utilization of the partograph, $61(48.4 \%)$ agreed to this factor determining their non-utilization of the partograph, $38(30.2 \%)$ disagreed, while $2(1.6 \%)$ strongly disagreed that this factor determines their nonutilization of the partograph. The mean response for this factor was $2.87(0.74) .47(37.3 \%)$ of the respondents strongly agreed that lack of recognition /motivation of workers determines their non-utilization of the partograph, 37(29.4\%) agreed, 20(15.9\%) disagreed to this factor determining their non-utilization of the partograph while 22(17.5\%) strongly disagreed. The mean response for this factor was $2.87(1.11) .65(51.6 \%)$ of the respondents strongly agreed that lack of standard institutional guideline/ protocol on the use of partograph determines their non-utilization of the partograph, 39(31\%) agreed that this factor determines their nonutilization of the partograph, 6(4.8\%) disagreed, while 16(12.7) strongly disagreed to this factor determining their non-utilization of the partograph. The mean response for this factor was 3.21(1.02). 102(81\%) of the respondents strongly agreed that lack of training of staff through organization of seminars/ workshops on partograph in the health care facilities determines their non-utilization of the partograph, 24(19\%) agreed to this factor, while none $(0 \%)$ neither disagreed nor strongly disagreed that this factor determines their non-utilization of the partograph. The mean response for this factor was 3.81(0.39). 
The above administrative factors were accepted when their mean value is compared with the decision rule of 2.50 since they are above 2.50. The factors include non-availability of resources such as observation tools, for example sphygnomanometer, fetoscope etc (3.32), non availability of partograph chart in the labour ward (3.65), shortage of staff (3.44), ineffective and inadequate supervision from immediate boss (2.87), lack of recognition /motivation of workers (2.87), lack of standard institutional guideline/ protocol on the use of partograph (3.21) and lack of training of staff through organization of seminars/ workshops on partograph in the health care facilities (3.81). In the overall, the administrative factors for the non- utilization of the partograph are with mean value of 3.31 .

\section{Discussion}

To assess client-centered factors that determines the non-utilization of the partograph. From the results in table 1, the subjects (nurses/ midwives) pointed out that one of the main client-centered factors that determines their non- utilization of the partograph includes women reporting late in labour at $9 \mathrm{~cm}$ to $10 \mathrm{~cm}$ cervical dilatation. This may probably be due to inadequate education of mothers on signs of true labour which mainly occurs when there is shortage of staff making the staff to overlook some duties and pay more attention to others. This finding is in accordance with the finding of Azendegbe et al, (2012) in their Surveys in Benin and Ethiopia which reported that health workers perspective about women coming late at the hospital nearly in second stage of labour ( $8-10 \mathrm{~cm}$ cervical dilation) is one of the factors that influence partograph use.

Also, the condition of the women on admission to the labour ward such as women for elective ceasarean section, women for emergency caesarean section, women with disease condition such as severe pregnancy induced hypertension, eclampsia or confirmed cephalo-pelvic disproportion and women with antepartum haemorrhage were identified as factors to the non- utilization of the partograph. This may probably be due to un-attendance by mothers to antenatal care during pregnancy or due to the health seeking behavior of the patients. Moreover, utilization of the partograph is dependent on the woman's condition.

This finding is in accordance with the finding of Azendegbe et al, (2012) in their Surveys in Benin and Ethiopia which reported also that the condition of the women on presentation to the labour ward determines the use or non- use of the partograph in monitoring labour progress.

The findings of this study revealed that the major administrative factors that influence utilization of the partograph as shown in table 4 above are non-availability of resources such as observation tools, for example sphygnomanometer, fetoscope etc and non- availability of partograph chart in the labour ward. A worker requires tools/ instruments to carry out his/ her work effectively, therefore, the probable reason for nonutilization of the partograph by nurses/ midwives as suggested by the above factor may be lack of support from the administrators or financial constraint on the part of administrators. Also, effort of the nurses/ midwives to persistently ask for their needs may be the contributing factor to the above. This finding is in line with the findings of Dujardin, Shamphleire, Sene and Nchiaye (2008) in their study on value of alert and action lines on the partograph which reported that lack of support from management in terms of providing the essential supplies and equipment including the provision of partograph charts for use by midwives are profound problems in the adoption and utilization of the partograph.

Another factor implicated by the respondents as determinant of their non- utilization of the partograph is shortage of staff. This implies non commitment on the part of the administrators to employ and provide human and material resources to ensure provision of quality care thus, their non- use of the partograph. This finding is in line with the findings of Qureshi, Sekadde-kigondu and Mutiso (2010) in their study on rapid assessment of partograph utilization in Kenya which reported that the poor usage of the partograph was attributed to staff shortage and lack of necessary supplies such as fetoscope and blood pressure machines. Furthermore, the finding is in line with the studies of MC-Authur, Bick and Knowles (2012), in their study in postnatal care: evidence and guideline for management at South England which found shortage of staff as one of the biggest constraints in most health institutions and reported that the few midwives on duty are faced with so much responsibilities on a shift that some important aspect of midwifery care are haphazardly done or not done at all.

Furthermore, lack of standard institutional guideline/ protocol on the use of partograph was reported by the respondents as determinants of their non- utilization of the partograph. This supports the finding of lack of in-service training of workers as management cannot organize training on what they do not approve of. This finding corresponds with the studies of Ogwang, karyabakabo and Rutebemberwa (2009) in their study involving observations; record reviews and interviewing of staff in Uganda which reported poor use of partograph during labour which is mainly affected by health input factors. The researchers recommended training of health workers on partograph use, provision of guidelines and adequate resources.

Also, lack of training of staff through organization of seminars/ workshops on partograph in the health care facilities was documented as one of the reasons that determine the non- utilization of the partograph by the respondents. This implies non commitment of the administrators to update their workers' knowledge and skill 
on partograph use or it may be as a result of lack of interest to be updated on the part of the nurses/ midwives. This finding is supported by the study of Medhanyie, Spigt, Dinant and Blanco (2011), in their study on knowledge and performance of Ethiopian health extension workers on antenatal and delivery care which observed inadequate use of partograph associated with lack of trained personnel.

Also, ineffective and inadequate supervision from immediate boss and lack of recognition /motivation of workers were identified as factors that contribute to the non- utilization of the partograph. This explains why there was no institutional guideline on partograph use. This finding corresponds with the findings of Opiah, Bola, Ekere and Monjok (2012) in their study on knowledge and utilization of the partograph among midwives in the Niger Delta region of Nigeria, their finding revealed lack of support from management system as factor that affects partograph use.

\section{Conclusion And Recommendation}

From the result it was concluded that major factors emanating from the clients that impede utilization of the partograph includes woman reporting late in labour, woman with disease condition such as severe pregnancy induced hypertension, or confirmed cephalo-pelvic disproportion and woman with antepartum haemorrhage. The major care center administrative factors that impede the utilization of the partograph by nurses includes non-availability of resources such as partograph in the labour ward, shortage of staff and lack of training of staff through organization of seminars/ workshops on partograph. It was recommended that thought patients present late and in some cases with complications nurses should carry out their duties following the emergency obstetric protocol using the partograph and that management should stand up for their responsibility by providing the needed materials in the labour ward.

\section{References}

[1]. Dujardin B., De-Schampheleire I., Sene H. \& Ndiaye F. (2012) value of the alert and action lines on the partogram. Lancet 339 (8805) 1336-1338

[2]. Fawole A., Adekanle D. and Hunyinbo K (2010) Utilization of Partograph in Primary Health Care in South Western Nigeria: Nigeria Journal of Clinical Practice 13(1):200-204.

[3]. Fawole A.O, Adekanle D. \& Hunyinbo K. (2008) Knowledge and utilization of the Partograph Among Obstetric Care givers in South West Nigeria. African Journal of Reproductive Health 12(1): 22-29.

[4]. Magon N. (2011) Partograph Revisited; International Journal of Clinical Cases Investigations, 3:1-6.

[5]. Maternal and Neonatal Health (2011).The partograph, an essential tool for decision-making during labour. Best practice United States Agency for International Development. Available at www. Mnh.jhpiego.org .

[6]. Mathai M. (2010).The partograph for the prevention of obstructed labour clinical obstetrics and gynaecology 52(2)256-269.

[7]. Mathibe Neke J.M. (2009): facilitation of midwifery students regarding utilization of a partograph; Africa journal of nursing and midwifery 11(1): 34-47.

[8]. Ogwang S., Karyabakabo Z. \& Rutebemberwa E. (2009). Assessment of partogram Use During Labour in Rujumbara Health Sub district, Uganda. African Journal of Health Sciences 9 (52): 27-34.

[9]. Okechukwu E., Adesegun A., Niyi O., Babalola A., Uche O.(2013) impact of training on use of partograph on material and perinatal outcome in peripheral health centres .J. Turk. Gynaecol. Obstet 102: 249-252.

[10]. Opiah M.,Bola O., Ekere J. \& Monjok E. (2012) knowledge and utilization of the partograph among midwives in the Niger Delta Region of Nigeria. African Journal of Reproductive Health 16(1) 125-132.

[11]. Qureshi Z.P., sekadde kigondu C. \& Mutiso S.M.(2010) Rapid assessment of partograph utilization in selected maternity units in kenya. East African Medical journal. Retrieved from http.//dx.doi.org /10.4314/eamj v 8716.63681 on 8/6/15.

[12]. Umezulike A.C.,Onah E., \& Okaro J.M.(2009) use of the partograph among medical personnel in Enugu, Nigeria. International journal of Gynaecology and obstetric 65 (2) 203-205.

[13]. Walker J. (2012) Report from the second 1CM /WHO/UNICEF. Pre-congress workshop on midwifery education. Action for safe mother hood. International Journal of Gynaecology and obstetrics 38:57-58. 